\title{
Coherent backscatter radar imaging in Brazil: large-scale waves in the bottomside F-region at the onset of equatorial spread $F$
}

\author{
F. S. Rodrigues ${ }^{1, *}$, D. L. Hysell ${ }^{1}$, and E. R. de Paula ${ }^{2}$ \\ ${ }^{1}$ Cornell University, Earth and Atmospheric Sciences, 2122 Snee Hall, Ithaca, NY, 14853, USA \\ ${ }^{2}$ Instituto Nacional de Pesquisas Espaciais (INPE), P.O. Box 515, São José dos Campos, SP, Brazil \\ *now at: ASTRA, 12703 Spectrum Drive 101, San Antonio, TX, 78249, USA
}

Received: 1 November 2007 - Revised: 8 April 2008 - Accepted: 13 May 2008 - Published: 21 October 2008

\begin{abstract}
The $30 \mathrm{MHz}$ coherent backscatter radar located at the equatorial observatory in São Luís, Brazil $\left(2.59^{\circ} \mathrm{S}\right.$, $44.21^{\circ} \mathrm{W},-2.35^{\circ}$ dip lat) has been upgraded to perform coherent backscatter radar imaging. The wide field-of-view of this radar makes it well suited for radar imaging studies of ionospheric irregularities. Radar imaging observations were made in support to the spread $F$ Experiment (SpreadFEx) campaign. This paper describes the system and imaging technique and presents results from a bottom-type layer that preceded fully-developed radar plumes on 25 October 2005. The radar imaging technique was able to resolve decakilometric structures within the bottom-type layer. These structures indicate the presence of large-scale waves $(\sim 35 \mathrm{~km})$ in the bottomside F-region with phases that are alternately stable and unstable to wind-driven gradient drift instabilities. The observations suggest that these waves can also cause the initial perturbation necessary to initiate the Generalized Rayleigh-Taylor instability leading to spread $F$. The electrodynamic conditions and the scale length of the bottom-type layer structures suggest that the waves were generated by the collisional shear instability. These results indicate that monitoring bottom-type layers may provide helpful diagnostics for spread $F$ forecasting.
\end{abstract}

Keywords. Ionosphere (Equatorial ionosphere; Ionospheric irregularities; Plasma waves and instabilities)

\section{Introduction}

Electron density irregularities in the equatorial ionosphere have been observed for over 70 years. These irregularidades were first detected by vertical ionospheric radio sounders (Berkner and Wells, 1934). Early studies showed that density irregularities are responsible for range and frequency spread

Correspondence to: F. S. Rodrigues

(frodrigues@astraspace.net) in ionograms. For historical reasons, the manifestation of these irregularities in a number of different types of sensors is refered to simply as equatorial "spread $F$ ". Observations have shown that electron density irregulaties with scale-sizes ranging from a few $\mathrm{cm}$ to hundreds of $\mathrm{km}$ can be detected during spread $F$ events. Typical equatorial spread $F$ events are first observed in postsunset hours in the bottomside Fregion at the magnetic equator. In some cases, large-scale ionospheric plasma depletions can reach the topside ionosphere. These large-scale spread $F$ events are responsible for signal disruption in satellite-based communication and navigation systems (e.g. Kintner et al., 2001).

The ionospheric collisional interchange instability, also refered to as the Generalized Rayleigh-Taylor (GRT) instability, operates in the bottomside equatorial F-region and is usually invoked to explain the generation of plasma irregularities in the equatorial ionosphere (Kelley, 1989). Even though favorable conditions for instability growth can be found in the equatorial ionosphere during postsunset hours, an initial perturbation in the Pedersen conductivity is necessary to trigger spread $F$ in view of the relatively modest growth-rate of the instability. Atmospheric gravity waves are often invoked to explain the existence of such perturbations. Theories propose that gravity wave wind fields can directly modulate the electron density in the bottomside Fregion (e.g. Kelley et al., 1981; Vadas and Fritts, 2004) or can modulate the conductivity of thin E-region layers at low latitudes creating perturbation electric field (e.g. Prakash, 1999). These electric fields then map along magnetic field lines to the equatorial bottomside F-region. Direct measurements of atmospheric winds in the lower thermosphere are rarely available, however, making it difficult to determine the actual role of the gravity waves in the seeding process.

In order to further investigate the role of gravity waves in seeding equatorial spread $F$, a multi-instrumented campaign of aeronomical observations was conducted in Brazil between September and November of 2005. The spread

Published by Copernicus Publications on behalf of the European Geosciences Union. 


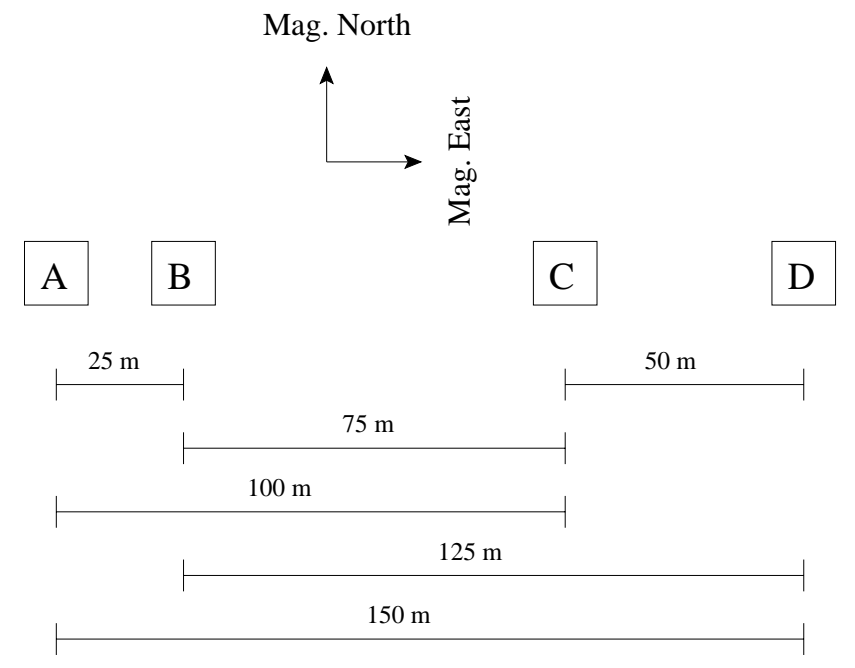

Fig. 1. Diagram showing the disposition of the four (A, B, C and D) antenna modules. The modules are aligned in the magnetic eastwest direction.

$F$ Experiment (SpreadFEx) campaign is part of an observational and theoretical collaborative effort between several universities and institutes in the US and Brazil. During the campaign, a number of instruments for aeronomical observations were operated simultaneously. The set of instruments included optical systems for nighttime airglow observations (photometers and all-sky imagers), dual frequencyreceivers GPS for total electron content (TEC) measurements, GPS-based scintillation monitors, and digisondes. These instruments were distributed at a number of locations over the Brazilian territory. Besides ground-based observations, satellite measurements made with the Global Ultraviolet Imager (GUVI) onboard the TIMED satellite and other instruments onboard the CHAMP satellite are planned to be used to characterize both the neutral atmosphere and the ionosphere during the campaign. The spreadFEx campaign not only allowed a multi-instrumented investigation of gravity wave activity and their impact on spread $F$ but also gave the opportunity to study other plasma plasma processes that can be connected with equatorial spread $F$.

The São Luís coherent backscatter radar made observations of F-region irregularities in support to the SpreadFEx campaign. This radar probes small-scale irregularities matching the Bragg condition for the operating frequency of $30 \mathrm{MHz}$. The radar has been making conventional coherent scatter radar and basic two-antenna interferometric observations of E- and F-region irregularidades since 2001. In 2005, the radar was upgraded, and two more antenna modules were added to the system. A total of four antenna modules became available, with the possibility of six nonredundant antenna baselines for interferometry purposes. Since then, the construction of in-beam radar images of the scattering layers has been possible.
In this paper, we discuss the signatures of a large-scale wave $(\sim 35 \mathrm{~km})$ in the radar images of a strong bottom-type layer detected on 25 October 2005 are examined. Bottomtype scattering layers presumably occur as frequently during the spread $F$ season in Brazil as they do over Jicamarca, in Peru. However, they are not so often observed with detectable signal-to-noise ratio (SNR) levels with the lowpower radar in São Luís, especially during periods of low solar flux. This makes radar imaging studies more difficult since the resolution of the images depend on the SNR of the echoes. The event of 25 October combined with the wide field-of-view of the São Luís radar gave us a rare opportunity to investigate the structure of bottom-type layers with a larger angular coverage than was previously possible in other radar imaging experiments (Hysell et al., 2004; Hysell and Chau, 2006).

\section{Experimental setup}

A $30 \mathrm{MHz}$ coherent backscatter radar is installed at the São Luís Observatory $\left(2.59^{\circ} \mathrm{S}, 44.21^{\circ} \mathrm{W},-2.35^{\circ}\right.$ dip lat $)$ in Brazil. This radar uses two $4-\mathrm{kW}$ peak-power transmitters for observations of small-scale (5-m) field-aligned electron density irregularities. Initially, only two antenna modules arranged side-by-side in the magnetic east-west direction were available for transmission and reception. Each antenna module is composed by an array of $4 \times 4$ Yagi antennas. With two antenna modules, only standard coherent backscatter observations and basic two-antenna interferometry were possible. Nevertheless, these two modules allowed real-time spread $F$ monitoring and measurements of the zonal as well as vertical drift velocity of the scattering structures at the magnetic equator in Brazil (e.g. de Paula and Hysell, 2004; de Paula et al., 2004; Rodrigues et al., 2004).

In order to perform radar imaging observations, two more antenna modules were added to the radar system in 2005 . Figure 1 shows a diagram with the disposition of the four antenna modules. The antenna modules are aligned with the magnetic east-west direction. Modules A and B in Fig. 1 are the original antenna modules and are used both for transmission and reception. Modules $\mathrm{C}$ and $\mathrm{D}$ are the new antenna modules and are used for reception only.

The set of four independent antenna modules can be used to perform interferometry with 6 nonredundant baselines, with baseline lengths varying from 25 to $150 \mathrm{~m}(2.5$ to $15 \lambda)$. The locations of the modules were chosen to give an approximately uniform distribution of baseline lengths.

Table 1 lists the main parameters for spread $F$ radar imaging observations in São Luís. Parameters for radar imaging experiments at Jicamarca are also shown for comparison purposes. Jicamarca is a much larger radar system and can provide higher resolution images. Usually at Jicamarca, imaging experiments use the north and south quarters of the main antenna for transmission and the modules (64ths of the main 
array) for reception. Each module is formed by $12 \times 12$ array of crossed dipoles.

\section{Interferometric radar imaging}

The use of radar interferometry in ionospheric studies was introduced by Woodman (1971). Farley et al. (1981) and Kudeki et al. (1981) used radar interferometry to study E- and F-region electron density irregularities, respectively. Kudeki and Sürücü (1991) generated the first interferometric images of equatorial electrojet irregularities. More recently, Hysell (1996) combined radar interferometry and the maximum entropy method to obtain higher resolution radar images.

\subsection{Inversion technique}

We have followed the approach described in Hysell (1996) to construct interferometric images of the scattering layers. Here, we give a summary of the technique, and refer the reader to Hysell (1996) and Hysell and Chau (2006) for a more complete mathematical derivation.

We start by expressing the mathematical relationship between the real valued function $f$ that describes the angular distribution of radar scatterers and the complex cross spectrum $g$ computed from signals arriving at two antennas spaced by a distance $d$ :

$g(k d, \omega)=\int \frac{d \psi}{\sqrt{1-\psi^{2}}} f(\psi, \omega) A(\psi) e^{i k d \psi}$

Here, $k$ is the radar wavenumber, $d$ is the antenna separation, which in our case is taken to be perpendicular to the magnetic meridian, $\omega$ is the Doppler frequency, $\psi$ is approximately the zenith angle in the magnetic equatorial plane, and $A(\psi)$ is a function representing the antenna pattern in the magnetic east-west direction. Adopting the notation used in Astronomy and followed by Hysell (1996), we refer to $g$ as the visibility function and $f$ as the brightness distribution. Equation (1) shows that $f$ and $g$ are related by an expression similar to a continuous spatial Fourier transform.

In radar imaging, we seek $f(\psi, \omega)$, which is the true angular distribution of the Doppler shifted backscattered signals in each radar range gate and for each incoherent integration period. With $n$ antenna modules available, it is possible to obtain estimates of $g$ for $M=n(n-1) / 2$ nonredundant baselines. Generally, $g$ is incompletely sampled, and finding $f$ given a limited, noisy data-set becomes a typical example of an inverse problem.

To obtain an estimate of $f(\omega, \psi)$, the maximum entropy (MaxEnt) approach for image reconstruction is used. With this technique, one searches for the brighntess distribution that maximizes the Shannon (or information) entropy constrained by the measurements and their uncertainties. The
Table 1. Radar parameters for equatorial spread $F$ imaging observations. Jicamarca parameters are also shown for comparison purposes (Hysell et al., 2004).

\begin{tabular}{lll}
\hline Parameter & São Luís & Jicamarca \\
\hline Peak power & $8 \mathrm{~kW}$ & $100 \mathrm{~kW}-1 \mathrm{MW}$ \\
Number of baselines & 6 & 28 \\
Baud length & $2.5 \mathrm{~km}$ & $300 \mathrm{~m}$ \\
Code length & $28 \mathrm{bits}$ & $28 \mathrm{bits}$ \\
Image angular coverage & $\sim 16^{\circ}$ & $\sim 8^{\circ}$ \\
Image angular resolution & $0.4^{\circ}$ & $0.1^{\circ}$ \\
\hline
\end{tabular}

discrete version of the Shannon entropy of the brightness distribution is given by:

$S=-\sum_{i} f_{i} \ln \left(f_{i} / F\right)$

where, $F=\sum_{i} f_{i}$ is the total brightness. Maximizing the entropy is equivalent to finding the brightness that is the most likely to have occurred in a random process. In image reconstruction, the entropy can be interpreted as a smoothness measure.

Given $M$ non-redundant interferometric baselines, it is possible to obtain $2 M+1$ independent measurements of the visibility. These data points correspond to the real and imaginary parts of a component of $g$ plus the absolute power. Therefore, for each baseline with a separation distance $d_{j}$, there is a measurement $g_{j}$ with a random uncertainty $e_{j}$ added to it. The relationship between the visibility and brightness can be written as:

$g_{j}+e_{j}=\sum_{i} f_{i} h_{i j}$

where $h_{i j}$ is either the real or the imaginary part of the interferometer point spread function $e^{j k d_{j} \psi_{i}}$. The brightness distribution that maximizes $S$ while being constrained by the data is the extremum of the functional:

$$
\begin{aligned}
E\left[f\left(\lambda_{j}\right)\right]=S+\sum_{j} \lambda_{j}\left(g_{j}+e_{j}\right. & \left.-\sum_{i} f_{i} h_{i j}\right) \\
& +\sum_{j} \Lambda\left(e_{j}^{2} \sigma_{j}^{-2}-\Sigma\right)
\end{aligned}
$$

where $\lambda_{j}$ are lagrange multipliers enforcing data constraints and $\Lambda$ is another lagrange multiplier enforcing a constraint on the error norm $\Sigma_{j}$. The $\sigma_{j}^{2}$ are theoretical error variances. Error covariances are neglected here. Maximizing a simpler version of Eq. (4) with respect to $f_{i}$ and another Lagrange multiplier introduced to enforce the normalization of the brightness yields a parameterized expression in $\lambda_{j}(\mathrm{Hy}-$ sell and Chau, 2006):

$f_{i}=F \frac{e^{-\sum_{j} \lambda_{j} h_{i j}}}{\sum_{i} e^{-\sum_{j} \lambda_{j} h_{i j}}}$ 
Maximizing Eq. (4) with respect to $\lambda_{j}$ yields $2 M+1$ nonlinear equations given by:

$g_{j}+e_{j}-\sum_{i} f_{i} h_{i j}=0$

These equations are solved numerically for the $2 M+1$ lagrange multipliers $\lambda_{j}$. Maximizing Eq. (4) with respect to $e_{j}$ yelds an expression relating the statistical fluctuations $e_{j}$ to the Lagrange multipliers $\lambda_{j}$.

$\lambda_{j}+\frac{2 \Lambda}{\sigma_{j}^{2}} e_{j}=0$

Finally, maximizing the functional with respect to $\Lambda$ yelds one more equation relating it to $\Sigma, \lambda_{j}$ and $\sigma_{j}^{2}$ :

$\Lambda^{2}-\sum_{j} \frac{\lambda_{j}^{2} \sigma_{j}^{2}}{4 \Sigma}=0$

\subsection{In-beam radar images}

Images are constructed from the spectral brightness distributions $f(\omega, \psi)$ for each range gate. Each spectral estimate is composed of four spectral bins. Colors are associated to each spectral bin. Green is used for the zero-frequency component, magenta for the Nyquist frequency component, and red and blue for the red- and blue-shifted components, respectively.

For each spectral bin, an image with the respective color associated with the bin is created. The intensity of the color in each pixel is proportional to the signal-to-noise ratio (SNR) of the spectral bin. The four images are added to produce the composite radar images presented in this paper. While pure color images indicate narrow spectral features, color combinations denote broad spectra. White color indicates flat (white) spectra. Conventional Range-TimeIntensity (RTI) maps can be formed by integrating the radar images over all frequencies and zenith angles. The angular coverage of the in-beam radar images is controlled by the radiation patterns of the transmitting and receiving antennas and by the dynamic range of the received echoes. For the São Luís radar, the field-of-view is approximately $16^{\circ}$ wide. The angular field-of-view also limits the maximum horizontal scale-size of the waves that can be unambiguosly observed. For the São Luís radar, the maximum scale is approximately $50 \mathrm{~km}$ at F-region heights.

\section{Radar observations}

Coherent backscatter radar observations were made almost every night during the SpreadFEx campaign from 25 September to 8 November 2005. The spread $F$ season in Brazil starts around mid-September and lasts until the end of April (Sobral et al., 2002; Abdu et al., 2003). RTI maps and range-time maps of the vertical and zonal velocities could be produced for every observation night using measurements made with only a pair of antenna modules. However, a reduced number of observations could be used for the generation of in-beam radar images due to a technical problem with one of the receiver channels. Primary attention has been given to observations made during the period of 24-27 October 2005, when good sky conditions for airglow measurements were found and preliminary results from a number of different observation instruments indicated the occurence of spread $F$ (Fritts et al., 2008). This paper focuses on the night of 25 October 2005 when a strong bottom-type layer was detected and radar images could be generated. Bottom-type layers are not so frequently observed at São Luís as they are at Jicamarca. This is, at least in part, due to the lower sensitivity of the radar in Brazil.

Figure 2 shows the RTI map for 25 October. The RTI map shows that first detected echoes come from a thin scattering layer. The layer is approximately $35 \mathrm{~km}$ thick and starts around $250 \mathrm{~km}$ altitude. This thin layer was observed between 21:45 UT and 22:15 UT. The RTI map also shows that at least three fully developed radar plumes occurred later in the night. The first plume was observed around 22:15 UT and reached about $500 \mathrm{~km}$ in altitude. A second plume was observed around 23:30 UT and reached over $700 \mathrm{~km}$. A third plume was observed around 01:30 UT and also reached over $700 \mathrm{~km}$. Echoes for this third plume were much weaker and indicate that small-scales waves responsible for radar echoes were decaying. RTI maps are ambiguous indicators of the space-and-time distribution of the scattering irregularities. This is due to the "slit camera" distortion effect, which is caused by the finite beamwidth of the radar antenna and the motion of the plasma across and along the radar field-of-view during the development of the radar plume. Structures with horizontal scale-sizes of tens of kilometers cannot be distinguised in the RTI maps, hence the necessity of interferometric techniques for radar studies of spread $F$.

Using the MaxEnt algorithm, in-beam images of the spread $F$ event of 25 October were constructed. Figure 3 shows a sequence of radar images between 21:45 UT and 21:55 UT, when a thin scattering layer is seen in the RTI map. The radar images show how echoes actually come from a localized structure that emerged to the east (right) of the radar site and moved westward entering the radar field-ofview. The initial westward velocity of the structure was about $80 \mathrm{~m} / \mathrm{s}$ and decreased as time passed. This westward moving scattering structure was located in the bottomside F-region, probably in the valley region. Another sequence of images from the period between 22:06 UT and 22:08 UT is shown in Fig. 4. These images show a second scattering structure entering the field-of-view of the radar on the east side as the first structure moved to the west. Radar imaging shows that what seems to be a homogeneous scattering layer in the RTI map is actually highly structured in the horizontal direction. The fact that a scattering cluster is within the radar beam at 


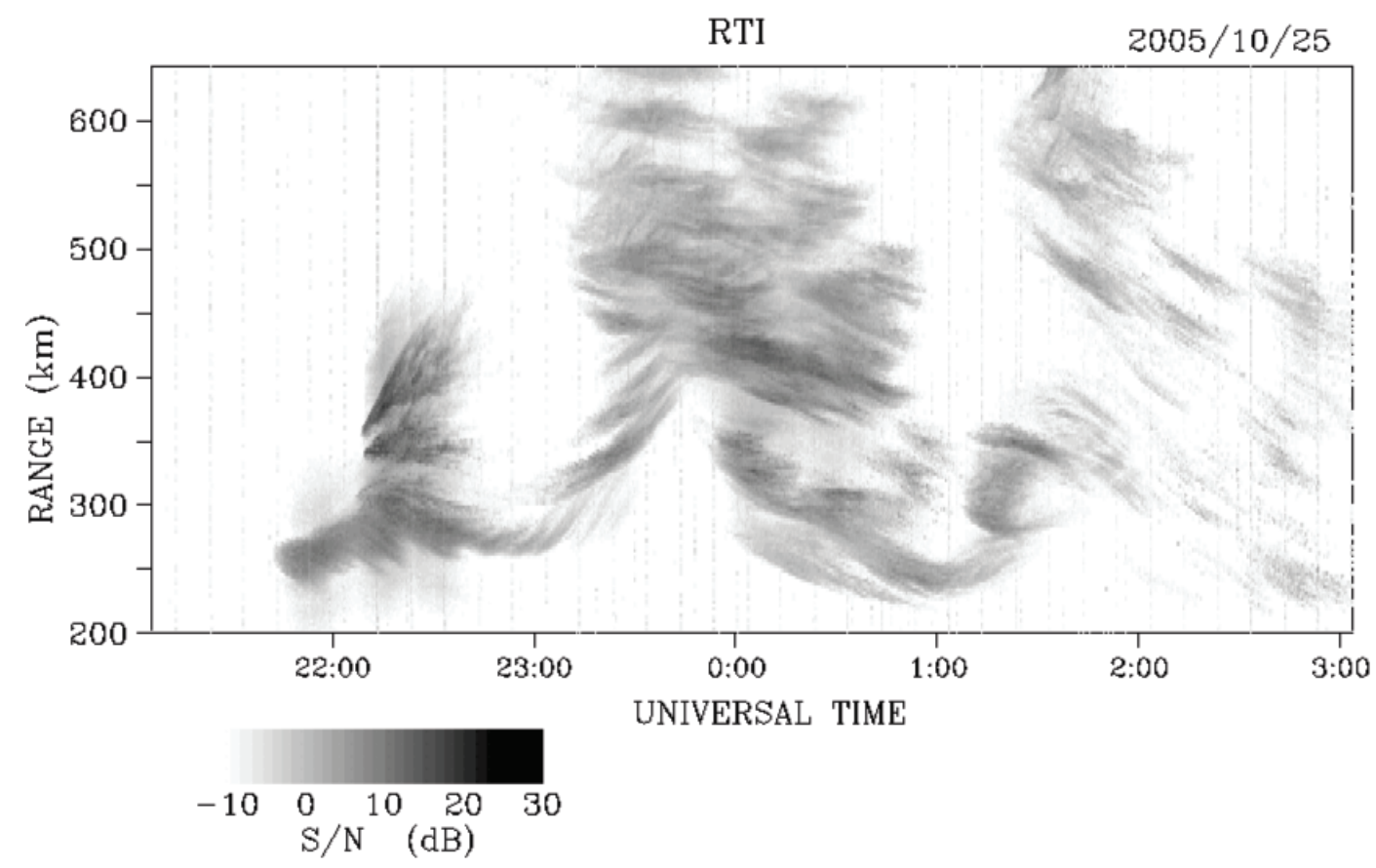

Fig. 2. Range-Time-Intensity (RTI) map for 25 October 2005 (UT $\approx$ LT+3)

any given time and the "slit camera" effect mentioned earlier cause these structures to appear as homogeneous layers in the RTI maps. Horizontal modulation of scattering layers in the bottomside F-region was observed at other nights, but the organization of the scattering structures in the images was not as clear as in the case of 25 October. A third sequence of images is shown in Fig. 5. This sequence shows images from the period between 22:08 UT and 22:16 UT. It shows that the scattering clusters continue to move, slowly now, in the westward direction. The images also show that scattering structures start to emerge at higher ranges. As the initial scattering clusters move westward, a vertically elongated scattering structure develops, reaching F-peak altitudes. This scattering structure corresponds to the bottomside radar plume seen in the RTI map around 22:15 UT created via GRT instability.

\section{Discussion}

\subsection{Bottom-type layers}

The characteristics of the layer shown in Fig. 3 match those of bottom-type scattering layers frequently observed by the Jicamarca radar in Peru during spread $F$ season. Bottomtype layers are vertically narrow bands of scattering irregularities in the bottomside F-region moving westward or slowly eastward (Hysell and Burcham, 1998a). These layers occur after sunset and do not develop vertically. They are precursors of radar plumes, which are signatures in the RTI maps of large-scale ionospheric plasma depletions generated via GRT instability.

A better understanding of the generation of bottom-type layers came from the development of an advanced observation mode at Jicamarca Observatory that combines incoherent and coherent backscatter radar measurements (Kudeki et al., 1999; Kudeki and Bhattacharyya, 1999). Kudeki and Bhattacharyya (1999) used this mode to observe the so-called post-sunset plasma vortex in the equatorial region. Their observations showed that the strata where the plasma motion is retrograde with respect to neutral wind and where bottomtype layers are located would be unstable to wind-driven gradient drift instabilities, given that horizontal density gradients are present. These gradients would be generated by defragmentation of the plasma vortex. Gradient drift instabilities can have growth rates for intermediate-scale waves much larger than the maximum predicted for the GRT instability for typical differential plasma-neutral flow speeds. Smallscale plasma waves responsible for radar scattering would be generated presumably by the primary waves via three-wave mode coupling (Hysell et al., 2004).

Observations also showed that the plasma vortex is stronger when the pre-reversal enhancements of the vertical plasma drift is larger, and this would also explain why bottom-type layers share the climatology of equatorial spread $F$ (Kudeki et al., 1999). Radar imaging observations contributed, showing that bottom-type layers are formed by kilometric structures with characteristics matching those 



Fig. 3. Sequence of in-beam radar images showing a bottom-type layer. The images shows that the layer is created to east of the radar location and moves in westward direction entering the radar field-of-view.

expected for irregularities created by wind-driven gradient drift instability (Hysell et al., 2004). In-situ measurements of the vertical electric field measurements made by rockets confirmed the predominance of structures with vertical scalesizes of a few $\mathrm{km}$ within bottom-type layers (Hysell et al., 2006). Due to the vertical resolution $(2.5 \mathrm{~km})$ of the measurements made with the São Luís radar, the primary kilometric waves within the bottom-type structure could not be distinguised in the images as they can be at Jicamarca.

\subsection{Vertical shear of the zonal plasma flow}

By looking at the zonal displacement of the scattering structure in the sequence of radar images of Fig. 3 one can notice the westward motion of the structure. It is also possible to see that irregularities at lower ranges move faster. The initial westward velocity at lower ranges was about $80 \mathrm{~m} / \mathrm{s}$. Because of the inconstant zonal velocity with altitude, the structure distorts and tilts to the east.

The westward motion of bottom-type layers is a feature that has been commonly observed at Jicamarca using interferometric techniques (e.g. Kudeki et al., 1981). Bottomtype irregularities move with the background plasma and indicate the presence of a vertical shear in the zonal flow of the plasma at F-region heights. The shear is formed by ionospheric plasma at F-region peak heights moving in the eastward direction with the thermospheric wind, and bottomside F-region plasma moving in the westward direction (e.g. Fejer, 1981; Tsunoda et al., 1981; Haerendel et al., 1992).

Zonal drifts are altitude independent in the equatorial Fregion except during periods of fast drift variations and at times around the pre-reversal enhancement (Fejer, 1981).
While first studies indicated the action of both E- and Fregion dynamos as main drivers of the vertical shear (e.g. Heelis et al., 1974), recent results have shown that other factors can be potentially important as well. These factors would include zonal electric fields on flux tubes with significant Hall conductivity, vertical winds, and vertical boundary currents forced from above or below the flux tube in question (Haerendel et al., 1992; Hysell et al., 2005a).

\subsection{Large-scale waves and spread $F$}

The sequence of in-beam radar images in Fig. 3 illustrates interesting features that can be detected with the Brazilian radar. Initially, only one cluster of scatterers was observed in the radar images. As the cluster moves westward (to the left), another cluster enters the field-of-view of the radar. These horizontally spaced irregularity clusters suggest the presence of large-scale horizontal plasma waves in the bottomside Fregion. These waves would create regions with horizontal density gradients that are alternately stable and unstable to wind-driven gradient drift instabilities. The spacing length between scattering clusters $(\sim 35 \mathrm{~km})$ would correspond to the wavelength of the large-scale wave.

The plasma wave responsible for modulating the bottomtype layer can also have served as the seed wave for the GRT instability leading to fully developed radar plumes. Figure 5 shows a rare observation, where a sequence of radar images shows the full development of a radar plume that occurred within the radar field-of-view. The plume starts to develop in the region between and above the two bottom-type clusters. As the bottom-type clusters continue to move westward, the radar plume evolves vertically and starts to move slowly 

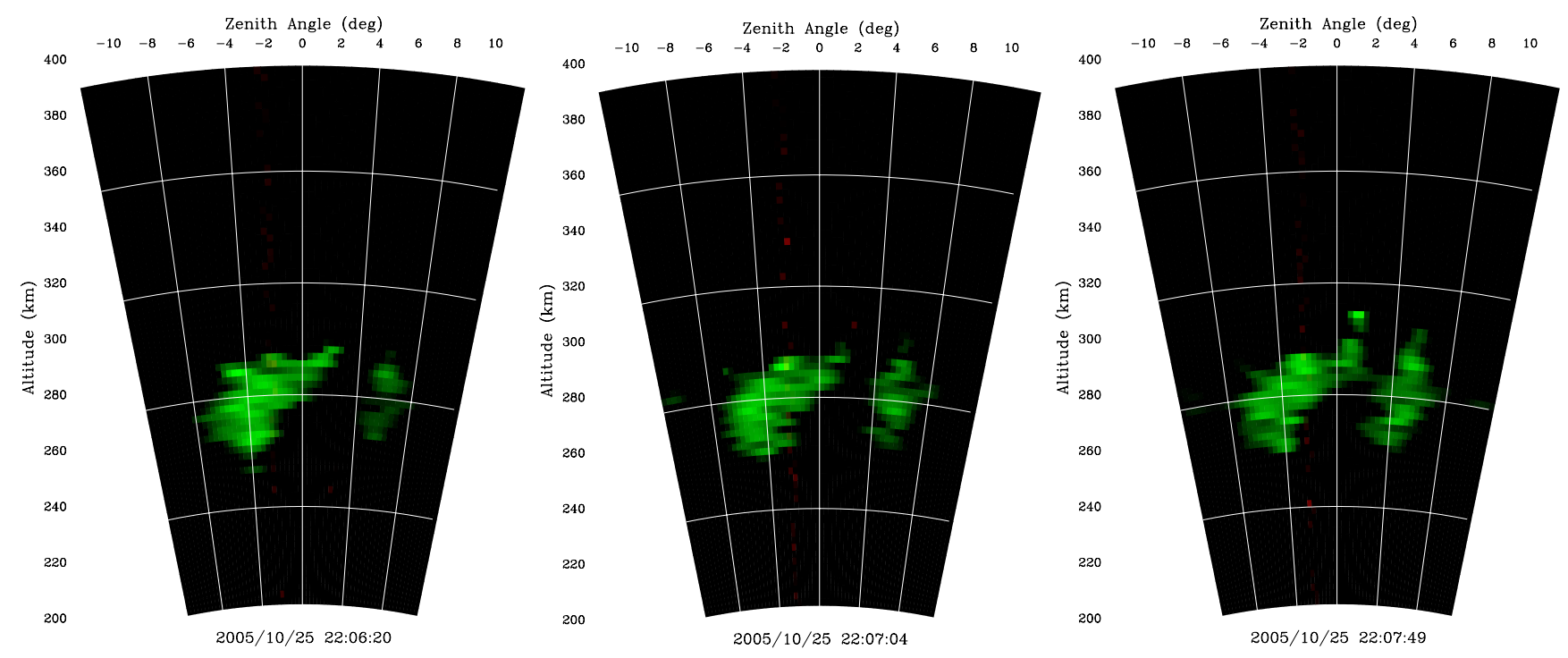

Fig. 4. Sequence of in-beam radar images showing two clusters of scatterers within the radar field-of-view.


Fig. 5. Sequence of in-beam radar images showing the development of a radar plume.

eastward. The radar images indicate that the two different and independent types of instability could be consequences of the same large-scale wave.

\subsection{Previous observations}

The radar imaging observations at São Luís confirm the presence of decakilometric horizontal structures previously detected at Jicamarca. Hysell et al. (2004) observed clusters of scattering irregularities horizontally spaced by approximately $30 \mathrm{~km}$ in bottom-type layers. The structures observed at Jicamarca were located around $330 \mathrm{~km}$ altitude, while the structures observed in São Luís were located around $270 \mathrm{~km}$. This difference in altitude is within the height variability of the bottom-type layers. In both cases, the structures span about $40 \mathrm{~km}$ altitude and were moving westward. In the Jicamarca case, no tilt in the structures could be observed. The spacing between the clusters observed in São Luís is slightly larger than the spacing of the structures observed at Jicamarca. The short duration of the bottom-type layer on 25 October allowed the detection of only two clusters of scattering structures before the development of the radar plume. 
Clusters of bottom-type layers spaced by a $30-50 \mathrm{~km}$ were also detected by the ALTAIR radar (Hysell et al., 2005b). Clusters of bottom-type layers spaced by $150-250 \mathrm{~km}$ were also found in ALTAIR observations. The radar also showed that electron density undulations with similar scale-lengths (tens of $\mathrm{km}$ or $150-250 \mathrm{~km}$ ) in the bottomside F-region accompanied the scattering clusters. Scattering clusters have only been observed in bottom-type layers that preceded fully developed radar plumes. Horizontal structures in bottomtype layers have not been observed at nights when spread $F$ did not occur (Hysell et al., 2004). This suggests that monitoring such structures would provide a practical diagnostic for spread $F$ forecasting.

\subsection{On the origin of the large-scale waves}

Hysell and Kudeki (2004) investigated the possibility of shear flow to generate large-scale plasma waves in the bottomside equatorial ionosphere. They found a collisional branch of the electrostatic Kelvin-Helmholtz instability that could operate under typical conditions of the bottomside equatorial F-region. The main destabiling factor for this collisional shear instability is the retrograde motion of plasma with respect to the neutral wind, commonly observed in the bottomside equatorial F-region.

Nonlocal boundary value analysis of the collsional shear instability reported by Hysell and Kudeki (2004) predicted larger growth rates for wave modes with wavelengths $(\lambda)$ in the order of $4 \pi L$, where $L$ is the length scale of the shear flow for a vertical profile of the zonal velocity $v(z)$ modelled as $v(z)=v_{o}(z) \tanh (z / L)$. Their results showed that the growth rate for these modes is comparable to growth rates of the collisional interchange instability in the equatorial F-region under ideal conditions (4-5 e-foldings per hour). Hysell and Kudeki (2004) also pointed out using a time-dependent numerical simulation of the shear instability that waves with shorter wavelengths of order $L$ are observed in the initial stage of the instability development. The growth rate for these transient modes, however, was relatively long ( $\sim 1$ e-folding per hour). More recently, Kudeki et al. (2008) revisited the collisional shear instability with a nonlocal linear analysis. Their results showed larger growth rates for the $\lambda=L$ mode (18 e-foldings per hour), while the results for $\lambda=4 \pi L$ agreed with the results obtained in Hysell and Kudeki (2004).

The wavelengths $(\lambda \sim L)$ predicted by the collisional shear instability would explain why periodic structures observed in the bottomside ionosphere preferably have scale lengths of a few tens of $\mathrm{km}$. Electron density undulations with wavelenghts of a few hundreds of $\mathrm{km}$, often observed with the ALTAIR radar could also be explained in terms of the longer wavelengths modes $(\lambda \sim 4 \pi L)$ with slower growth rates. Another possibility is the action of $100-300 \mathrm{~km}$ gravity waves that may reach the lower F-region (Vadas, 2007). The amplitudes of these waves and their impact in the ionospheric plasma is the subject of an ongoing theoretical study that is part of the SpreadFEx campaign.

The observations at São Luís suggest that the large-scale wave modulating the bottom-type layer on 25 October was created by the collisional shear instability. A strong vertical shear in the zonal velocity is implied by the westward motion of the layer at lower F-region heights. This indicates the appropriate conditions for the collisional shear instability to develop. The spacing between the bottom-type structures is also in agreement with the scale lengths of fastest growing waves predicted by the instability theory (Hysell and Kudeki, 2004; Kudeki et al., 2008). The scale length also matches previous imaging radar observations made at Jicamarca (Hysell et al., 2004) and ALTAIR radar observations in Kwajalein (Hysell et al., 2005b, 2006).

\section{Conclusions}

We have presented in-beam radar images of F-region scattering layers using interferometric observations made with the $30 \mathrm{MHz}$ radar in Brazil. The new set of antenna modules added to the system allows the study of spread $F$ structures with resolution of a few kilometers. This paper focused on the analysis of a bottom-type layer detected on 25 October 2005 during the SpreadFex campaign. Other interesting features observed in the scattering layers over São Luís will be discussed in future reports.

The finite width of the radar field-of-view makes the bottom-type irregularities appear as a continuous layer in the RTI maps. The interferometric radar images for 25 October confirmed the observations of Hysell et al. (2004) showing that bottom-type layers can be formed by clusters of scattering irregulatities. This indicates the presence of a large-scale plasma wave in the bottomside F-region. The plasma wave modulates the bottomside F-region, creating regions that are alternately unstable and stable to wind-driven gradient drift instabilities. The images also suggest that the same largescale wave could have served as a seed wave for the GRT instability, leading to a radar plume.

The observations made with the São Luís radar are additional experimental evidence of the presence of large-scale waves in the bottomside F-region. While the possibility of gravity waves being responsible for the large-scale wave structure in the radar images cannot be ruled out, the strong shear in the zonal plasma flow and the scale length of the wave structure suggest its generation by the collisional shear instabiliy (Hysell and Kudeki, 2004; Kudeki et al., 2008). Also, periodic structures in bottom-type layers with similar scale lengths were previously observed at other equatorial sites (Hysell et al., 2004, 2006), suggesting a common and somewhat deterministic mechanism of generation.

Regardless of the origin of these waves, monitoring largescale horizontal structures in the bottom-type scattering layers provides a useful diagnostic for the forecast of equatorial 
spread $F$. First attempts to track these structures using interferometric observations at Jicamarca have failed (D. L. Hysell, personal communication), mainly because of the narrow field-of-view of the antenna. The observations made in São Luís are encouraging. They indicate that these large-scale structures can be detected with small, low power radar systems. Similar systems could be easily deployed at other observation sites.

Acknowledgements. The authors would like to thank Acácio Cunha Neto for operating the São Luís radar and Lázaro Camargo for pre-processing the radar data. The São Luís radar was partially supported by FAPESP Processes 99/00026-0 and 04/01065-0.

Topical Editor M. Pinnock thanks J. L. Chau and S. Vadas for their help in evaluating this paper.

\section{References}

Abdu, M. A., Souza, J. R., Batista, I. S., and Sobral, J. H. A.: Equatorial spread F statistics and empirical representation for IRI: A regional model for the Brazilian longitude sector, Adv. Space Res., 31, 703-716, 2003.

Berkner, L. V. and Wells, H. W.: F-region ionosphere - investigation at low latitudes, Terr. Magn., 39, 215-230, 1934.

de Paula, E. R. and Hysell, D. L.: The São Luis $30 \mathrm{MHz}$ coherent scatter ionospheric radar: System description and results, Radio Sci., 39, RS1014, doi:10.1029/2003RS002914, 2004.

de Paula, E. R., Iyer, K. N., Hysell, D. L., Rodrigues, F. S., Kherani, E. A., Jardim, A. C., Rezende, L. F., Dutra, S. G., and Trivedi, N. B.: Multi-technique investigations of storm-time ionospheric irregularities over the São Luís equatorial station in Brazil, Ann. Geophys., 22, 3513-3522, 2004,

http://www.ann-geophys.net/22/3513/2004/.

Farley, D. T., Ierkic, H. M., and Fejer, B. G.: A new technique for studying plasma turbulence in the ionosphere, J. Geophys. Res., 86, 1467-1472, 1981.

Fejer, B. G.: The equatorial ionospheric electric fields: A review, J. Atmos. Terr. Phys., 43, 377-386, 1981.

Fritts, D. C., Abdu, M. A., Batista, B. R., Batista, I. S., Batista, P. P., Buriti, R., Clemesha, B. R., Comberiate, J., Dautermann, T., de Paula, E., Fechine, B. J., Fejer, B., Gobbi, D., Haase, J., Kamalabadi, F., Laughman, B., Lima, P. P., Liu, H., Medeiros, A., Pautet, D., Sao Sabbas, F. S., Sobral, J. H. A., Stamus, P., Takahashi, H., Taylor, M. J., Vadas, S. L., and Wrasse, C.: The Spread F Experiment (SpreadFEx): Program overview and first results, Earth, Planets Space, in press, 2008.

Haerendel, G., Eccles, J. V., and Cakir, S.: Theory for modeling the equatorial evening ionosphere an the origin of the shear in horizontal plasma flow, J. Geophys. Res., 97, 1209-1223, 1992.

Heelis, R. A., Kendal, P. C., Moffet, R. J., Windle, D. W., and Rishbet, H.: Electrical coupling of the $\mathrm{E}$ and $\mathrm{F}$ regions and its effect on F region drifts and winds, Planet. Space Sci., 22, 743-756, 1974.

Hysell, D. L.: Radar imaging of equatorial F region irregularities with maximum entropy interferometry, Radio Sci., 31, 15671578, 1996.

Hysell, D. L. and Burcham, J. D.: JULIA radar studies of equatorial spread-F, J. Geophys. Res., 103, 29 155-29 167, 1998a.
Hysell, D. L. and Chau, J. L.: Optimal aperture synthesis radar imaging, Radio Sci., 41, RS2003, doi:10.1029/2005RS003383, 2006.

Hysell, D. L. and Kudeki, E.: Collisional shear instability in the equatorial F region ionosphere, J. Geophys. Res., 109, A11301, doi:10.1029/2004JA010636, 2004.

Hysell, D. L., Chun, J., and Chau, J. L.: Bottom-type scattering layers and equatorial spread-F, Ann. Geophys., 22, 4061-4069, 2004 ,

http://www.ann-geophys.net/22/4061/2004/.

Hysell, D. L., Kudeki, E., and Chau, J. L.: Possible ionospheric preconditioning by shear flow leading to equatorial spread F, Ann. Geophys., 23, 2647-2655, 2005a, http://www.ann-geophys.net/23/2647/2005/.

Hysell, D. L., Larsen, M. F., Swenson, C. M., Barjatya, A., Wheeler, T. F., Sarango, M. F., Woodman, R. F., and Chau, J. L.: Onset conditions for equatorial spread $\mathrm{F}$ determined during EQUIS II, Geophys. Res. Lett., 32, L24104, doi:10.1029/2005GL024743, $2005 b$.

Hysell, D. L., Larsen, M. F., Swenson, C. M., Barjatya, A., Wheeler, T. F., Bullett, T. W., Sarango, M. F., Woodman, R. F., Chau, J. L., and Sponseller, D.: Rocket and radar investigation of background electrodynamics and bottom-type scattering layers at the onset of equatorial spread F, Ann. Geophys., 24, 1387-1400, 2006, http://www.ann-geophys.net/24/1387/2006/.

Kelley, M. C.: The Earth's Ionosphere, Academic Press, San Diego, Calif., 487 pp., 1989.

Kelley, M. C., Larsen, M. F., Lahoz, C., and McClure, J. P.: Gravity wave initiation of equatorial spread F - A case study, J. Geophys. Res., 86, 9087-9100, 1981.

Kintner, P. M., Kil, H., Beach, T. L., and de Paula, E. R.: Fading timescales associated with GPS signals and potential consequences, Radio Sci., 36, 731-744, doi:10.1029/1999RS002310, 2001.

Kudeki, E. and Bhattacharyya, S.: Postsunset vortex in equatorial F-region plasma drifts and implications for bottomside Spread-F, J. Geophys. Res., 28, 28 163-28 170, 1999.

Kudeki, E. and Sürücü, F.: Radar interferometric imaging of field aligned plasma irregularities in the equatorial electrojet, Geophys. Res. Lett., 18, 41-44, 1991.

Kudeki, E., Fejer, B. G., Farley, D. T., and Ierkic, H. M.: Interferometer studies of equatorial $\mathrm{F}$ region irregularities and drifts, Geophys. Res. Lett., 8, 377-380, 1981.

Kudeki, E., Bhattacharyya, S., and Woodman, R. F.: A new approach in incoherent scatter $\mathrm{F}$ region $\boldsymbol{E} \times \boldsymbol{B}$ drift measurements at Jicamarca, J. Geophys. Res., 28, 28 145-28 162, 1999.

Kudeki, E., Akgiray, A., Milla, M., Chau, J. L., and Hysell, D. L.: Equatorial spread-F initiation: Post-sunset vortex, thermospheric winds, gravity waves, J. Atmos. Sol.-Terr. Phys., 69(1718), 2416-2427, 2008.

Prakash, S.: Production of electric field perturbations by gravity wave winds in the $\mathrm{E}$ region suitable for initiating equatorial spread F, J. Geophys. Res., 104, 10 051-10 070, doi:10.1029/ 1999JA900028, 1999.

Rodrigues, F. S., de Paula, E. R., Abdu, M. A., Jardim, A. C., Iyer, K. N., Kintner, P. M., and Hysell, D. L.: Equatorial spread F irregularity characteristics over São Luís, Brazil, Radio Sci., 39, RS1S31, doi:10.1029/2002RS002826, 2004.

Sobral, J. H. A., Abdu, M. A., Takahashi, H., Taylor, M. J., de Paula, 
E. R., Zamlutti, C. J., de Aquino, M. G., and Borba, G. L.: Ionospheric plasma bubble climatology over Brazil based on 22 years (1977-1998) of $630 \mathrm{~nm}$ airglow observations, J. Atmos. Sol. Terr. Phys., 64, 1517-1524, 2002.

Tsunoda, R. T., Livingstone, R. C., and Rino, C. L.: Evidence of a velocity shear in bulk plasma motion associated with the postsunset rise of the equatorial F layer, Geophys. Res. Lett., 8, 807810, 1981.

Vadas, S. L.: Horizontal and vertical propagation and dissipation of gravity waves in the thermosphere from lower atmospheric and thermospheric sources, J. Geophys. Res., 112, A06305, doi:10.1029/2006JA011845, 2007.
Vadas, S. L. and Fritts, D. C.: Thermospheric responses to gravity waves arising from mesoscale convective complexes, J. Atmos. Sol. Terr. Phys., 66, 781-804, 2004.

Woodman, R. F.: Inclination of the geomagnetic field measured by an incoherent scatter technique, J. Geophys. Res., 76, 178-184, 1971. 